\title{
Correction to: Trauma and Resilience Among Displaced Populations
}

\section{Correction to:}

G. Theisen-Womersley, Trauma and Resilience Among

\section{Displaced Populations,} https://doi.org/10.1007/978-3-030-67712-1

The original version of this book was inadvertently published without open-access funder information. This has been updated in the book. 